\title{
Prognostic value of functional tumor burden on 68Ga-DOTATOC PET/CT in patients with pancreatic neuro-endocrine tumors
}

\author{
J. OHNONA ${ }^{1}$, V. NATAF ${ }^{1}$, M. GAUTHÉ ${ }^{1}$, S. BALOGOVA ${ }^{1,2, *}$, O. BÉLISSANT BENESTY ${ }^{1}$, J. ZHANG-YIN ${ }^{1}$, J-N. TALBOT ${ }^{1}$, F. MONTRAVERS ${ }^{1}$ \\ ${ }^{1}$ Hôpitaux Universitaires Est Parisien, AP-HP Hôpital TENON, Department of Nuclear Medicine and Université Sorbonne, Paris, France; \\ ${ }^{2}$ Department of Nuclear Medicine, Faculty of Medicine, Comenius University and St. Elisabeth Oncology Institute, Bratislava, Slovakia
}

${ }^{*}$ Correspondence: sona.balogova@ousa.sk

Received March 28, 2018 / Accepted June 8, 2018

\begin{abstract}
Despite their relative quiescence, pancreatic neuro-endocrine tumors (pNET) can correspond to various presentations and outcomes. Several prognostic factors have been identified, including maximal standardized uptake value of the most intense focus ( $m a x S U V_{\max }$ ) on Positron Emission Tomography (PET) with a somatostatin analogue. Herein, we investigate the prognostic value on progression free-survival of the total functional tumor volume (TFTV) measured by ${ }^{68}$ Ga-DOTATOC PET. From patients who underwent ${ }^{68} \mathrm{Ga}$-DOTATOC PET from 2008 to 2014, we selected consecutive patients with G1 or G2 pNET (2010 World Health Organization classification), at least one abnormal focus on PET and available follow-up data. TFTV was computed by summing the volumes of all pathological foci, delineated use of $41 \%$ of its SUV $_{\max }$ for each threshold focus. Fifty patients were included. During the follow-up period, 33 patients had stable or responsive disease (66\%; median duration 28.5 months; range 6.3-77.7 months) and 17 patients experienced disease progression (34\%; median progression time 21 months; range 6.7-44.7 months). Median PFS was 43.5 months. The best TFTV cut-off for predicting progression within 24 months was $13.8 \mathrm{~cm}^{3}$. Multivariate analysis determined that TFTV greater than $13.8 \mathrm{~cm}^{3}$ was the only criterion considered a significant risk factor for tumor progression (HR 2.9; $\mathrm{p}=0.0003$ ). A significant difference in PFS was observed for TFTV ( $<13.8$ vs. $\geq 13.8 \mathrm{~cm}^{3}$ : median not reached vs. 25 months; $\mathrm{p}=0.0001$ ).

Our study suggests that $68 \mathrm{Ga}$-DOTATOC TFTV measured on PET images is a valuable prognostic biomarker in patients with well-differentiated pNETs of all stages.
\end{abstract}

Key words: ${ }^{68} \mathrm{Ga}$-DOTATOC, positron emission tomography, pancreatic neuroendocrine tumors, functional tumor volume, prognostic biomarker

Pancreatic neuro-endocrine tumors (pNET) are rare, with an estimated incidence less than 1 in 100,000 [1]. Compared to cancers of the exocrine pancreas, pNETs are slowly progressive, have relatively good prognosis, and can have a variety of clinical presentations and outcomes [2, 3]. Identifying prognostic markers is therefore crucial for the management of these patients. Tumor grade [4] and disease extent [5] have already been recognized as relevant prognostic factors.

PET imaging using ${ }^{68} \mathrm{Ga}$-labeled somatostatin analogues is progressively replacing ${ }^{111} \mathrm{In}$-pentetreotide scintigraphy as first-line imaging method for diagnosis, staging and restaging of pNETs $[6,7]$. This recent imaging modality is a powerful tool in the management of patients with well-differentiated neuro-endocrine tumors (NET) [7]. One of its various advantages over scintigraphy and SPECT is the possibility of performing semi-quantitative analysis.
Ambrosini et al. [3] assessed the prognostic value of maximum standardized uptake value of the most intense abnormal focus (maxSUV $\max$ ) on ${ }^{68} \mathrm{Ga}$-DOTANOC PET in patients with pNETs and a higher $\mathrm{SUV}_{\max }$ was associated with better outcome.

Recent measurements of tumor burden by metabolic tumor volume (MTV) have been thoroughly reported in the use of ${ }^{18} \mathrm{~F}$-fluorodeoxyglucose (FDG) PET/CT in lymphoma and then in a wide range of solid tumors. The prognostic value on overall survival of MTV at initial staging has been demonstrated [8-10].

The diagnostic target of ${ }^{68} \mathrm{Ga}-\mathrm{DOTATOC}$ is not a metabolic pathway, but the pathological over-expression of somatostatin receptors (mainly type 2 and 5). Therefore, we propose the term functional tumor volume (FVT) for the evaluation of tumor volume showing pathologically increased 
somatostatin receptor expression and total functional tumor volume (TFVT) for the summed lesion volume at whole body level. To the best of our knowledge, no study has been published on the prognostic value of functional tumor volume measurements in patients with NETs on PET/CT using a ${ }^{68} \mathrm{Ga}$-labelled somatostatin analogue.

Herein, we investigate the prognostic value on progression-free survival (PFS) of tumor burden in patients with pNETs. This is evaluated by means of the total functional tumor volume (TFTV) which over-expresses somatostatin receptors (SSR), as shown on ${ }^{68} \mathrm{Ga}$-DOTATOC PET. Our secondary objective is to check whether our series confirm the results of Ambrosini et al. [3] on the prognostic value of $\operatorname{maxSUV}_{\max }$ on PFS in patients with pNETs.

\section{Patients and methods}

Patients. With these objectives, we reviewed the data of the patients referred to our department for ${ }^{68} \mathrm{Ga}$-DOTATOC PET/CT examination between September 2008 and December 2014 and patients were included in the prospective study from September 2008 to September 2012. The study is "PHRC DOTATOC" EUDRACT 2007-002610-19 and individual authorization was obtained from the French Medicine Agency (ANSM) for each patient from October 2012. All patients gave their informed consent. We selected the data of all consecutive patients with histologically proven G1 or G2 pNET, according to the 2010 World Health Organization classification [4]. The additional inclusion criteria in the present analysis were the detection of at least one abnormal focus on ${ }^{68} \mathrm{Ga}$-DOTATOC PET/CT and available follow-up data after PET/CT.

All patients underwent physical examination at inclusion by each referring physician in departments specialized in the management of neuroendocrine tumors. Clinical history was recorded to identify the presence of symptoms induced by functioning tumors.

Follow-up visits were performed by the referring physician after ${ }^{68} \mathrm{Ga}$-DOTATOC PET/CT; imaging follow-up included CT at 6 months (+/- one month) after inclusion. Progression was assessed on CT using the RECIST criteria [11]. If progression was suspected clinically or biologically but not confirmed on conventional imaging, another ${ }^{68} \mathrm{Ga}$-DOTATOC PET/CT was performed to check for appearance of new pathological foci. This was considered disease progression, similarly to the current RECIST 1.1 criteria using FDG PET [11].

Patient management was decided by each referring physician according to the general status of the patient, the stage of the disease and the results of PET/CT or of liver MRI. The type of management was recorded: surgery, medical therapy (non-radioactive somatostatin analogues, chemotherapy or targeted therapy), somatostatin receptor radionuclide therapy (SRRT), local therapy of a single metastatic site or therapeutic abstention.
The protocol for this ancillary study was approved by the local Ethics Committee and the study was performed in compliance with the Helsinki Declaration for human studies.

PET/CT imaging. ${ }^{68} \mathrm{Ga}$-DOTATOC was prepared in the radio-pharmacy of our Nuclear Medicine department. ${ }^{68} \mathrm{Ga}$ was eluted from a ${ }^{68} \mathrm{Ge} /{ }^{68} \mathrm{Ga}$ generator and DOTATOC was labelled following the Breeman procedure [12]. The ${ }^{68} \mathrm{Ge} /{ }^{68} \mathrm{Ga}$ generator and the DOTATOC vials were both purchased from Iason $\mathrm{GmbH}$ (Graz, Austria). PET/CT with ${ }^{68} \mathrm{Ga}$-DOTATOC was performed after an intravenous injection of $2 \mathrm{MBq} / \mathrm{kg}$ of body mass of the radiotracer followed by an uptake phase of $60 \mathrm{~min}$. No fasting was needed prior administration.

${ }^{68} \mathrm{Ga}$-DOTATOC PET/CT acquisition covering a field of view from the skull to mid-thighs was performed on a Gemini TF 16 PET/CT machine (Philips Medical System). The CT acquisition was performed first (120 kVp $80 \mathrm{~mA} . \mathrm{s})$, and then the PET acquisition in $2 \mathrm{~min}$ per bed position in $3 \mathrm{D}$ mode with the time-of-flight determination.

Functional tumor volume computation. Any focus with a visual intensity greater than background that could not be explained by physiologic uptake or elimination (pituitary gland, spleen, liver, adrenals, uncinate process, kidneys and urinary tract) was considered pathological somatostatin receptor over-expression.

Total functional tumor volume (TFTV) of all visible lesions at whole body level was measured by a senior nuclear medicine physician on the attenuation-corrected images by summing up the functional volumes of all visible pathological ${ }^{68} \mathrm{Ga}$-DOTATOC foci. The limit of the functional volume of each focus was determined by applying a threshold of $41 \%$ of its $\mathrm{SUV}_{\max }$, using semi-automatic software (Tumor tracking, IntelliSpace Portal Philips Healthcare). This threshold of $41 \%$ was selected arbitrarily, but based on MTV data obtained by FDG $[9,13,14]$.

A spherical volume of interest (VOI) was drawn around each pathological uptake focus, and the functional volume was computed by summing all voxels above the threshold within the VOI.

$\mathrm{SUV}_{\max }$ was calculated by measuring the maximal concentration of ${ }^{68} \mathrm{Ga}$-DOTATOC in the region of interest corrected for body weight and injected activity $\left(\mathrm{SUV}_{\max }=\right.$ maximum activity concentration/[injected activity /body mass]) [15]. The highest $\mathrm{SUV}_{\max }$, corresponded to the most intense voxel of all VOIs, and the noted maxSUV $V_{\max }$, was recorded for each patient.

Data collection. Collected data on demographic, clinical and pathological features was recorded on a created computer sheet. The tumors were classified according to the 2010 WHO classification [4] and the TNM classification grading system [5]. The Ki-67 proliferation index was expressed as the percentage of Ki-67-positive cells in 2,000 tumor cells in the most highly immune-staining areas using the MIB1 antibody. 
Statistical analysis. Analysis was performed using dedicated software (Medcalc version 11.6.1.0; Windows). Median values and the respective ranges were used to describe the quantitative data. Groups were compared using the Pearson Chi-squared test, the Fisher's exact test for unpaired qualitative variables and the Mann-Whitney U test for unpaired continuous variables.

The number of pathological foci per patients was recorded and a histogram was created.

To evaluate the potential prognostic value of TFTV, we calculated the progression-free survival (PFS) rate by dividing the series of patients, based on disease status (progressive vs. stable disease) at the arbitrarily selected time point of 24 months after the ${ }^{68} \mathrm{Ga}$-DOTATOC PET/CT [3] . The PFS was defined as the time interval between ${ }^{68} \mathrm{Ga}$-DOTATOC PET/ $\mathrm{CT}$ and the time of disease progression. The area under the Receiver-Operating Characteristics curve (AUC) characterized the accuracy of the TFTV at baseline in predicting further disease progression at 24 months after PET/CT. The best prognosis cut-off value was estimated by the maximumlikelihood-ratio method.

PFS was also analyzed by the Kaplan-Meier method according to qualitative variables and the progression-free survival curves were compared using the log-rank test. The predictive risk factors for disease progression were determined by univariate and multivariate analyses using the Cox proportional hazards method. Risk factors were expressed as hazard ratios and $95 \%$ confidence intervals were determined.
The multivariate model was constructed using the backward method after all variables had been included and p-value was considered significant when less than 0.05 .

Kaplan-Meier, univariate and multivariate analyses for $\operatorname{maxSUV}_{\max }$ and TFTV were performed on the whole population and on two subgroups of the cohort: localized disease (stages I and II) and advanced disease (stages III and IV). For the maxSUV $V_{\max }$, the cut-off value of 37.8 was applied because this was previously established in the study of Ambrosini et al. [3]. No published threshold was available for TFTV, so we chose the cut-off values determined by ROC analysis.

\section{Results}

Patient characteristics. A total of 50 patients (26 men and 24 women; median age, $51 \mathrm{y} ; 20-77)$ with pNETs matched the criteria of the study. ${ }^{68} \mathrm{Ga}$-DOTATOC PET images were acquired after intravenous injection of an average $127 \mathrm{MBq}(51-182 \mathrm{MBq}) ; 1.8 \mathrm{MBq} / \mathrm{kg}$ of body mass $(0.7-2.4 \mathrm{MBq} / \mathrm{kg})$ and an uptake phase between administration of ${ }^{68} \mathrm{Ga}$-DOTATOC and average image acquisition of 58 min (40-120 min).

Eight patients (16\%) had functioning tumors: 4 insulinomas, 3 gastrinomas, and one glucagonoma; and 42 patients (84\%) non-functioning pNET. Eight patients had multiple endocrine neoplasia syndrome type 1 (MEN 1). According to the 2010 WHO classification, the pNET grade was G1 in 26 patients (52\%) and G2 in $24(48 \%)$.

Table 1. Patient characteristics in the entire population and stratified according to total functional volume

\begin{tabular}{|c|c|c|c|c|c|c|}
\hline \multirow{2}{*}{ Patient characteristic } & \multirow{2}{*}{$\begin{array}{c}\text { No. of } \\
\text { patients }\end{array}$} & \multicolumn{3}{|c|}{ Total functional tumor volume $\left(\mathrm{cm}^{3}\right)$} & \multirow{2}{*}{$\begin{array}{l}\text { Low tumor burden } \\
\text { TFTV }<13.8(\mathrm{n}=28)\end{array}$} & \multirow{2}{*}{$\begin{array}{l}\text { High tumor burden } \\
\text { TFTV } \geq 13.8(n=22)\end{array}$} \\
\hline & & Median & Range & p-value & & \\
\hline \multicolumn{7}{|l|}{ Gender } \\
\hline Male & 26 & 22 & $2.1-601$ & & 11 & 15 \\
\hline Female & 24 & 10.9 & $1.7-370$ & $\mathrm{p}=0.25$ & 17 & 7 \\
\hline \multicolumn{7}{|l|}{ Functioning NET } \\
\hline Yes & 8 & 6.5 & $2.3-24$ & & 7 & 1 \\
\hline No & 42 & 17.4 & $1.7-601$ & $\mathrm{p}=0.22$ & 21 & 21 \\
\hline \multicolumn{7}{|l|}{ Stage } \\
\hline I & 14 & 2.2 & $2.1-22$ & & 13 & 1 \\
\hline IIA & 6 & 7 & $2.5-25.4$ & & 5 & 1 \\
\hline IIIB & 9 & 3.8 & $1.7-48$ & & 7 & 2 \\
\hline IV & 21 & 50.4 & $9.6-601$ & & 3 & 18 \\
\hline Stages I/II & 20 & 3.2 & $2.1-25.4$ & & & \\
\hline Stages III/IV 2010 WHO classification & 30 & 22.8 & $1.7-601$ & $\mathrm{p}<0.0001$ & & \\
\hline NET G1 & 26 & 9.3 & $1.7-370$ & & 21 & 5 \\
\hline NET G2 & 24 & 24 & $2.3-601$ & $\mathrm{p}=0.0062$ & 7 & 17 \\
\hline \multicolumn{7}{|l|}{ Ki67 } \\
\hline$<=5 \%$ & 40 & 12.1 & $1.7-6.1$ & & 25 & 15 \\
\hline$>5 \%$ & 10 & 36.5 & $2.5-186$ & $\mathrm{p}=0.21$ & 3 & 7 \\
\hline \multicolumn{7}{|l|}{ Status at $24 \mathrm{mo}$ (40 patients analysed) } \\
\hline Stable disease & 29 & 26 & $1.7-271$ & & & \\
\hline Progressive disease & 11 & 165 & $4.5-601$ & $\mathrm{p}<0.001$ & & \\
\hline
\end{tabular}


The median Ki-67 index of the NET tumor was $2.5 \%$ (1-16\%); less than or equal to $5 \%$ in 40 patients, and greater than $5 \%$ in 10 patients (20\%). According to the TNM classification, the disease stage was I in 14 patients (28\%), IIA in 6 patients (12\%), IIIB in 9 patients (18\%) and IV in 21 patients (42\%). The subgroup of patients with stages III and IV disease included 30 patients and the subgroup of stages I and II 20 patients. The characteristics of all 50 patients are listed in Table 1.

${ }^{68} \mathrm{Ga}$-DOTATOC foci and total functional tumor volume (TFTV). The number of pathological foci per patient ranged from 1 to 51 (median: 3); the histogram for the distribution of the number of foci per patient is displayed in Figure 1. Data on median TFTV and the respective ranges among the different groups of patients are reported in Table 1. TFTV differed significantly according to the stage (stage III/IV vs. I/II, median: $22.8 \mathrm{~cm}^{3}$ vs. $\left.3.2 \mathrm{~cm}^{3} ; \mathrm{p}<0.0001\right)$, the 2010 WHO classification (NET G2 vs. NET G1: $24 \mathrm{~cm}^{3} \mathrm{vs} .9 .3 \mathrm{~cm}^{3}$; $\mathrm{p}=0.0062$ ) and the clinical status at 24 months of follow-up (progressive disease vs. stable disease: $165 \mathrm{~cm}^{3}$ vs. $26 \mathrm{~cm}^{3}$; $\mathrm{p}<0.001)$. There were no significant differences with respect to gender, the presence of tumor syndrome or Ki 67 using a cut-off value of $5 \%$.

Follow-up after ${ }^{68} \mathrm{Ga}-\mathrm{DOTATOC}$ PET/CT. The 50 patients were followed up for a median of 25.6 months (6.3-77.7 mo). Of the 50 patients, 19 (38\%) received medical therapy alone (non-radioactive somatostatin analogues, chemotherapy or targeted therapy), 2 patients received SRRT (4\%), 18 patients (36\%) underwent surgery, 6 patients $(12 \%)$ underwent surgery and received long-lasting somatostatin analogues and 2 patients (4\%) had a local treatment (radiofrequency of a single macroscopic metastasis in the liver); therapeutic abstention was decided for 3 patients (6\%).

PFS rate at 24 months. PFS rate at 24 months of followup was $73 \%(29 / 40)$. Ten patients were not included in this analysis because they had stable disease and a followup period of less than 24 months. As reported in Table 1, pre-treatment TFTV was significantly higher $(\mathrm{p}<0.001)$ in patients who progressed within 24 months of follow-up (11 patients; median $165 \mathrm{~cm}^{3}$; range $4.5-601 \mathrm{~cm}^{3}$ ) than in patients with stable disease (29 patients; median $26 \mathrm{~cm}^{3}$; range $1.7-271 \mathrm{~cm}^{3}$ ).

The ROC analysis showed that baseline TFTV was quite accurate in predicting whether a patient will progress within 24 months after PET/CT (AUC \pm SE, 0.88 \pm 0.05 ; $<<0.0001$ ); the best cut-off volume was the summed volume of all lesions detected at whole body level with ${ }^{68} \mathrm{Ga}$-DOTATOC uptake intensity $>41 \%$ of $\mathrm{SUV}_{\max }$ in a given lesion. This was $13.8 \mathrm{~cm}^{3}$ with a sensitivity and specificity to predict further progression within 24 months of $88 \%$ and $82 \%$, respectively.

Predictors for tumor progression during the whole follow-up period. During the overall follow-up period, 33 patients had stable or responsive disease $(66 \%$; median duration $28.5 \mathrm{mo}$; range 6.3-77.7 mo; Figure 2) and 17 patients experienced disease progression (34\%; median time for progression $21 \mathrm{mo}$; 6.7-44.7 mo; Figure 3). Disease progression was assessed on ${ }^{68} \mathrm{Ga}$-DOTATOC PET/CT for 10 of the 17 patients and on conventional imaging (CT or MRI) for 7 patients.

By univariate analysis, the variables found to be predictive for tumor progression during the whole follow-up period are summarized in Table 2. Risk factors for tumor progression were a NET G2 vs. G1 (HR 1.5; p=0.01), a stage III or IV vs. a stage I or II (HR 1.6; $\mathrm{p}=0.03)$, and a TFTV greater than $13.8 \mathrm{~cm}^{3}$ (HR 2.33; $\mathrm{p}=0.002$ ).

By multivariate analysis, TFTV greater than $13.8 \mathrm{~cm}^{3}$ was the only significant risk factor (HR 2.9; $\mathrm{p}=0.0003$ ). In the subgroup of stage III or IV patients (30 patients), multivariate analysis also showed that TFTV greater than $13.8 \mathrm{~cm}^{3}$ was the only significant risk factor (HR 1.9 p=0.01).

Gender, functioning status, non-surgical vs. surgical treatment after PET/CT, MEN syndrome, Ki-67 greater than 5\%, maxSUV $_{\max }$ less than 37.8 were not risk factors for tumor progression; neither in the whole series nor in the subgroup of stage III or IV patients.

We applied TFTV and maxSUV $V_{\max }$ at baseline for the whole series and combined these to calculate sensitivity, specificity, positive and negative predictive values and accuracy for progressive status prediction (Tables 3 and 4).

Our study confirmed significant differences in PFS with Ki-67, using the cut-off value of $5 \%$ established by Panzuto et al. [2] and Ambrosini et al. [3] and the cut-off value of $2 \%$ corresponding to the 2010 WHO classification [4].

Analysis of PFS on the whole follow-up period for the entire population. Median PFS was 43.5 months (mo. Figure 4). Significant differences in PFS were observed for TFTV $\left(<13.8 \mathrm{~cm}^{3}\right.$ vs. $\geq 13.8 \mathrm{~cm}^{3}$ : median not reached vs. $25 \mathrm{mo} ; \mathrm{p}=0.0001$ ) (Figure 4 ), Ki-67 ( $<5 \%$ vs. $\geq 5 \%$ : $44.7 \mathrm{mo}$ vs. $33.1 \mathrm{mo}$; $=0.04$ ), the 2010 WHO classification (NET G1 vs. NET G2: 44.7 mo vs. $32.6 \mathrm{mo} ; \mathrm{p}=0.008$ ).

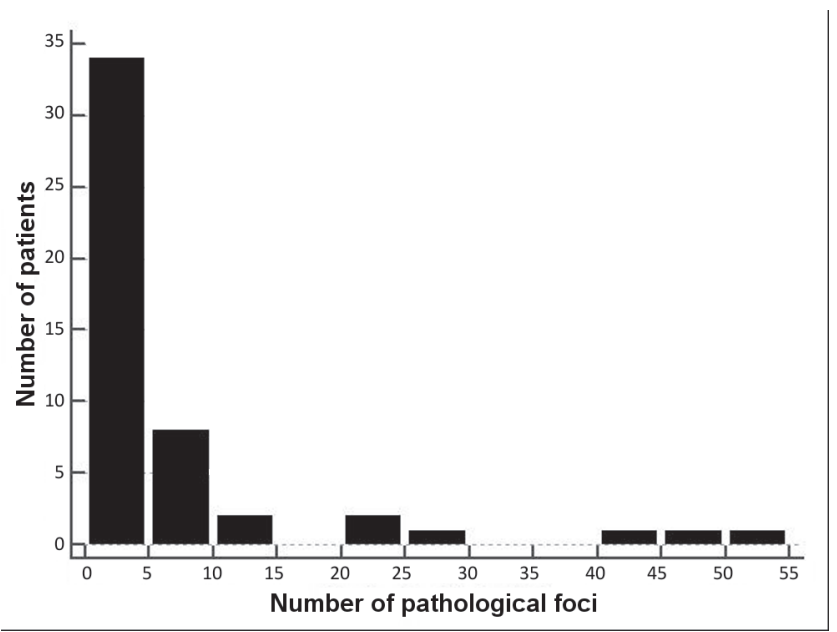

Figure 1. Histogram of the number of pathological foci detected by ${ }^{68} \mathrm{Ga}$ DOTATOC PET/CT per patient. 
A

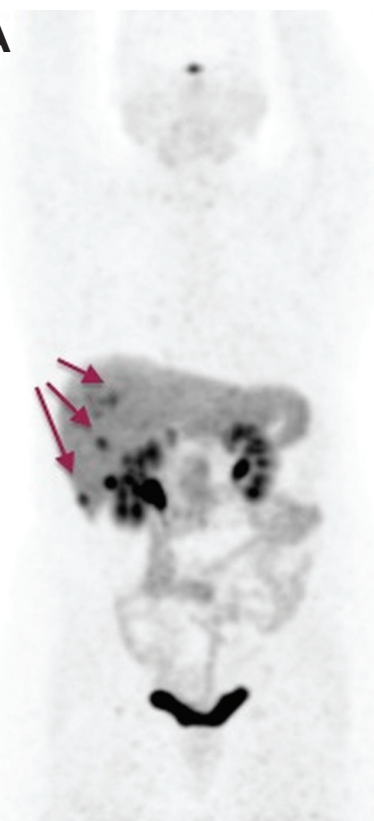

B

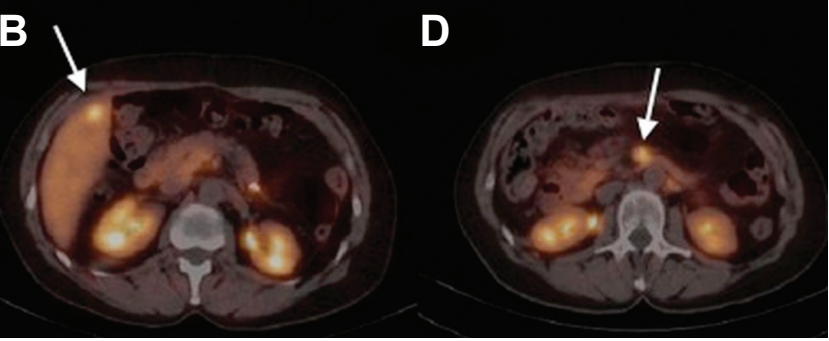

Figure 2. ${ }^{68} \mathrm{Ga}$-DOTATOC PET/CT of a 37-year-old male patient with pancreatic G1 NET. Maximum intensity projections (A and $C$ ) and transaxial slices (B and D). TFTV was $3.6 \mathrm{~cm}^{3}$ and $\operatorname{maxSUV}$ max 22 on baseline $\mathrm{PET} / \mathrm{CT}$ (A and B). Patient was treated with somatostatine analogues. Disease was stable at 20 mo of follow-up (C and D).
A

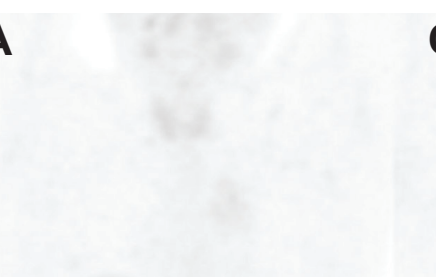

C
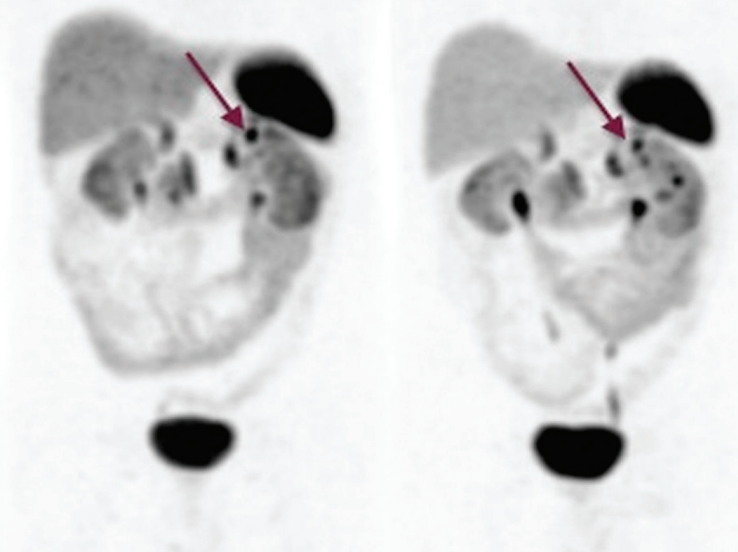

B

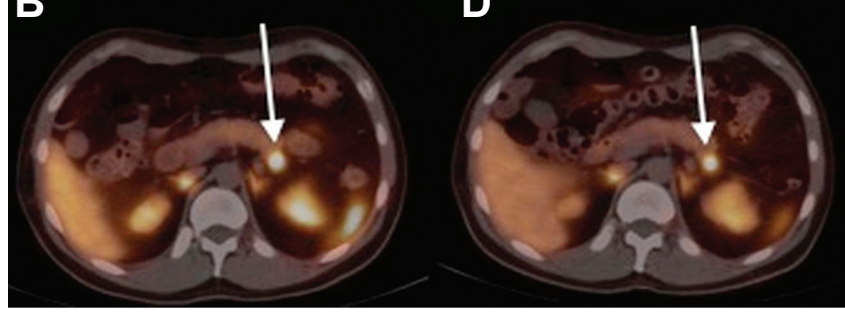

Figure 3: ${ }^{68} \mathrm{Ga}$-DOTATOC PET/CT in a 57-year-old female patient with pancreatic G2 NET and secondary hepatic lesions. Maximum intensity projections (A and $\mathrm{C}$ ) and transaxial slices (B and D). TFTV was $\mathbf{5 0 . 4}$ $\mathrm{cm}^{3}$ and $\operatorname{maxSUV} V_{\max } 8.8$ on baseline PET/CT (A and B). Patient underwent partial hepatectomy. Disease progressed at 19.1 mo of follow-up (C and $\mathrm{D}$ ) with the detection of a retropancreatic $68 \mathrm{Ga}$-DOTATOC positive lymph node.

Table 2. Risk factors for disease progression at 24 months, univariate and multivariate analyses

\begin{tabular}{|c|c|c|c|c|c|c|}
\hline \multirow{3}{*}{ Patient characteristics } & \multicolumn{6}{|c|}{ Analyses during the whole follow-up period } \\
\hline & \multicolumn{3}{|c|}{ Univariate } & \multicolumn{3}{|c|}{ Multivariate } \\
\hline & HR & 95\% confidence interval & p-value & HR & 95\% confidence interval & p-value \\
\hline Sex (male vs. female) & 14 & $0.34-2.47$ & $\mathrm{p}=0.88$ & - & - & - \\
\hline Neuro-endocrine syndrome (no vs. yes) & 0.89 & $0.05-3.1$ & $\mathrm{p}=0.39$ & - & - & - \\
\hline Stage I/II vs. III/IV & 1.6 & $1.1-21.9$ & $\mathrm{p}=0.03$ & - & - & - \\
\hline NET G2 vs. G1 & 1.5 & $1.3-16.1$ & $\mathrm{p}=0.01$ & - & - & - \\
\hline Treatment (non-surgical vs. surgery) & 0.42 & $0.55-4.14$ & $\mathrm{p}=0.4$ & - & - & - \\
\hline MEN vs. no MEN & 1.2 & $0.03-2.2$ & $\mathrm{p}=0.24$ & & & \\
\hline Ki67 (>5\% vs. $\leq 5)$ & 1.001 & $0.96-7.6$ & $\mathrm{p}=0.07$ & - & - & - \\
\hline Ki67 (continuous) & 13.9 & $33.26-42 \times 10^{9}$ & $\mathrm{p}=0.008$ & & & \\
\hline TFTV (>13.8 vs. $\leq 13.8$ ) & 2.33 & $2.3-45.6$ & $\mathrm{p}=0.002$ & 2.9 & $3.7-90.3$ & $\mathrm{p}=0.0003$ \\
\hline TFTV (continuous) & 125 & $1.004-1.01$ & $\mathrm{p}<0.0001$ & - & - & - \\
\hline $\operatorname{maxSUV}_{\max }(>37.8$ vs. $\leq 37.8)$ & 0.2 & $0.22-1.63$ & $\mathrm{p}=0.31$ & - & - & - \\
\hline $\operatorname{maxSUV} V_{\max }$ continuous & 0.003 & $0.99-1.01$ & $\mathrm{p}=0.61$ & - & - & - \\
\hline
\end{tabular}


The following "median not reached" classification indicates that more than half the patients were considered responding or stable at the end of the follow-up period. No significant difference was found by the $\operatorname{maxSUV}_{\max }(\geq 37.8 \mathrm{vs}$. <37.8: median not reached vs. $43.6 \mathrm{mo}$; $\mathrm{p}=0.35$ ), gender (men vs. women: 43.5 vs. $32.5 \mathrm{mo}$; $=0.88$ ), the functioning status (functioning vs. non-functioning: 43.5 mo vs. median not reached; $\mathrm{p}=0.3$ ), disease stage (I and II vs. III and IV: median not reached vs. $32.5 \mathrm{mo} ; \mathrm{p}=0.05$ ), type of treatment (surgery vs. other treatment : median not reached vs. $43.5 \mathrm{mo}$; $\mathrm{p}=0.4$ ), the presence of a MEN syndrome (MEN syndrome present vs. absent: median not reached vs. $43.5 \mathrm{mo} ; \mathrm{p}=0.21$ ). The threshold maxSUV $V_{\max }$ with significant prognostic value for patient's outcome at 24 months was not found.

PFS and multivariate analysis according to the stage of the disease. In the subgroup of 30 stage III or IV patients, 15 patients (50\%; median follow-up $28.5 \mathrm{mo}$; range $6.3-43.8 \mathrm{mo}$ ) had stable disease, and 15 patients had disease progression during the overall follow-up period (50\%; median time to progression $21 \mathrm{mo}$; range $6.7-44.7 \mathrm{mo}$ ). In this subgroup, as in the overall series, TFTV was quite accurate in predicting if a patient would progress (AUC \pm SE, $0.85 \pm 0.07 ; \mathrm{p}<0.0001$ ). The best cut-off value was $13.8 \mathrm{~cm}^{3}$ with a sensitivity and specificity for the prediction of tumor progression of $93 \%$ and $67 \%$ respectively.

In this subgroup, the median PFS was 24.5 months, and median not reached means that at the end of the follow-up period, more than half of patients were responding or stable. Significant differences in PFS were observed according to TFTV $\left(<13.8 \mathrm{~cm}^{3}\right.$ vs. $\geq 13.8 \mathrm{~cm}^{3}$ : median not reached vs.
$22.6 \mathrm{mo} ; \mathrm{p}=0.04)$ and the $2010 \mathrm{WHO}$ classification (NET G1 vs. NET G2: 44.7 mo vs. $32.5 \mathrm{mo} ; \mathrm{p}=0.02$ ). No significant difference was found according to the maxSUV $V_{\max }$ ( $<37.8$ vs. $\geq 37.8: 34$ mo vs. median not reached; $\mathrm{p}=0.76$ ), Ki-67 ( $<5 \%$ vs. $\geq 5 \%$ : 43.5 mo vs. 32.3 mo; $p=0.12$ ), gender (men vs. women: 43.5 vs. 32.5 mo; $\mathrm{p}=0.88$ ), the functioning status of the NET (functioning vs. non-functioning: $22.6 \mathrm{mo}$ vs. median not reached; $\mathrm{p}=0.32$ ), type of treatment (surgery

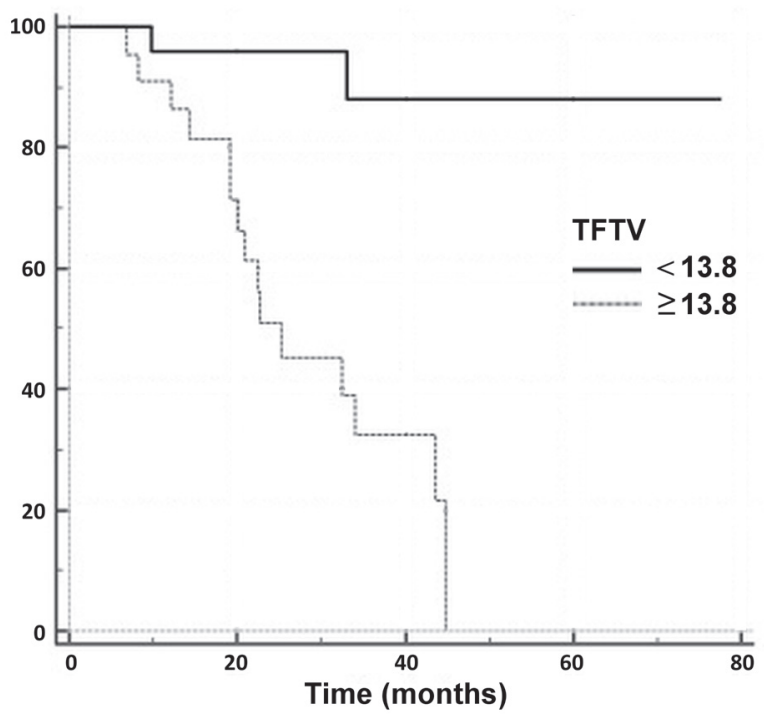

Figure 4. PFS in 50 patients with well-differentiated pNET (G1 and G2) according to baseline TFTV. PFS: progression free survival; total functional tumor volume; Solid line $=$ TFTV $<13.8 \mathrm{~cm}^{3}$; dashed line=TFTV $\geq 13.8 \mathrm{~cm}^{3} ; \mathrm{p}=0.0001$.

Table 3. Number of patients according to the progressive status, TFTV and the maxSUV $\max _{\max }$

\begin{tabular}{|c|c|c|c|c|c|c|}
\hline & \multicolumn{3}{|c|}{ Progressive disease (positive) } & \multicolumn{3}{|c|}{ Responsive or stable disease (negative) } \\
\hline & TTFV & TTFV & $\operatorname{maxSUV}_{\max }$ & TTFV & TTFV & $\operatorname{maxSUV}_{\max }$ \\
\hline & $\begin{array}{l}>13.8 \mathrm{~cm}^{3} \\
\text { (positive) }\end{array}$ & $\begin{array}{l}\leq 13.8 \mathrm{~cm}^{3} \\
\text { (negative) }\end{array}$ & & $\begin{array}{l}>13.8 \mathrm{~cm}^{3} \\
\text { (positive) }\end{array}$ & $\begin{array}{l}\leq 13.8 \mathrm{~cm}^{3} \\
\text { (negative) }\end{array}$ & \\
\hline $\operatorname{maxSUV}_{\max } \leq 37.8$ (positive) & 10 & 2 & 12 & 4 & 23 & 27 \\
\hline$(n=39)$ & TP both criteria & & TP & FP both criteria & & FP \\
\hline $\operatorname{maxSUV}_{\max }>37.8$ (negative) & 4 & 0 & 4 & 4 & 3 & 7 \\
\hline$(\mathrm{n}=11)$ & & FN both criteria & FN & & FN both criteria & $\mathrm{TN}$ \\
\hline 14 & & 2 & & 8 & 26 & \\
\hline TP TFTV & & FN TFTV & & FP TFTV & TN TFTV & \\
\hline$(n=16)$ & & & & & $(\mathrm{n}=34)$ & \\
\hline
\end{tabular}

TP: true positive; TN: true negative

Table 4. Diagnostic performance to predict progressive disease according baseline TFTV, maxSUV $\mathrm{max}_{\max }$ and combination of both criteria.

\begin{tabular}{lccc}
\hline Parameter & TFTV (positive if $>\mathbf{1 3 . 8}^{3}$ ) & maxSUV $_{\max }$ (positive if $<$ 37.8) & $\begin{array}{c}\text { TFTV and maxSUV }_{\max } \text { combined } \\
\text { (positive only if both criteria are positive) }\end{array}$ \\
\hline Sensitivity & $14 / 16=88 \%$ & $12 / 16=75 \%$ & $10 / 16=63 \%$ \\
Specificity & $26 / 34=77 \%$ & $7 / 34=21 \%$ & $30 / 34=88 \%$ \\
Positive predictive value & $14 / 22=58 \%$ & $12 / 39=31 \%$ & $10 / 14=71 \%$ \\
Negative predictive value & $26 / 28=93 \%$ & $7 / 11=64 \%$ & $30 / 36=83 \%$ \\
Accuracy & $40 / 50=80 \%$ & $19 / 50=38 \%$ & $40 / 50=80 \%$ \\
\hline
\end{tabular}


vs. other treatment: 22.4 mo vs. 33.1 mo; $\mathrm{p}=0.24$ ), or a MEN syndrome (present vs. absent: 30 mo vs. 29 mo; $\mathrm{p}=0.33$ ). The threshold $\operatorname{maxSUV} V_{\max }$ with significant prognostic value for patient's outcome at 24 months was not found.

In the stage I and II patient subgroup of 20 patients, 18 had stable disease (90\%; median follow-up 28.4 mo; range 6.7-77.5 mo), and the remaining 2 had disease progression during the overall follow-up period (10\%; median time for progression 9.8 and $25.2 \mathrm{mo}$ ). This subgroup was too imbalanced (only 2 patients with progressive disease) to perform complementary statistical analysis.

\section{Discussion}

To the best of our knowledge, this is the first study to establish that a high total functional tumor volume based on ${ }^{68} \mathrm{Ga}$-DOTATOC uptake can be an independent prognostic biomarker for pNET; and higher volume gives poorer outcome.

In the past two decades, semi-quantitative analysis in FDG PET imaging has helped identify prognostic biomarkers in a wide range of solid tumors and hematology malignancies, including NETs for which a high FDG uptake correlates with poor prognosis [16-18].

Numerous studies have been based on SUV $\mathrm{V}_{\max }$ measurements and this is considered a convenient and reproducible parameter. More recently, volumetric parameters have created great interest in nuclear medicine physicians. Indeed, unlike $S U V_{\max }$ or $\max S \mathrm{SV}_{\max }$, which derive information from one single voxel, the one with the highest radionuclide concentration per focus or in the whole patient, volumetric parameters reflect the entire tumor burden.

In a recent study involving 20 patients with pNET of all grades, Kim et al. [19] suggested that FDG metabolic tumor volume was an independent prognostic factor for overall survival, with high MTV predicting shorter survival.

Herein, we chose progression-free survival, and not overall survival, as the outcome because well-differentiated lower grade NETS frequently over-express somatostatin receptors but they progress slowly and do not show increased glucose metabolism. This choice was proven appropriate because no patients died during a median follow-up period of 25.6 months.

For well-differentiated NETs, which are often FDG-negative, PET with ${ }^{68} \mathrm{Ga}$-labelled-somatostatin analogue (SRPET) is a powerful tool for diagnosis, staging and restaging [20] and also for selecting patients eligible for somatostatin analogue therapy, including SRRT [21]. SRPET is now part of the diagnostic flowchart for pNET; gradually replacing ${ }^{111}$ In-pentetreotide scintigraphy and SPECT $[6,7]$.

The prognostic value of foci maxSUV $V_{\max }$ on SRPET has recently been investigated by Ambrosini et al. [3] in 43 patients with well-differentiated (WHO G1 and G2) stages III or IV pNETs. These authors found high ${ }^{68} \mathrm{Ga}$-DOTANOC
$\operatorname{maxSUV}_{\max }$ correlates with better outcome. Our study did not confirm this prognostic value of maxSUV $V_{\max }$ using the proposed 37.8 cut-off value, even when combined with TFTV to better reflect disease extension. Furthermore, the threshold maxSUV $V_{\max }$ with significant prognostic value for patient outcome at 24 months was not found in our study.

A further difference between the two studies is that the somatostatin analogue for PET/CT was DOTANOC in Ambrosini [3] and DOTATOC in our work. The different affinity profiles of the two analogues could explain the cut-off value of maxSUV $V_{\max }$ reported by those authors did not give different outcome in our study cohort. Furthermore, the study populations were not identical because we investigated all stage patients while Ambrosini et al only included stage III and IV patients [3]. Such advanced disease could be consistent with heterogeneous and partly less differentiated tumors. In this population, it is likely that a high maxSUV $V_{\max }$ reflecting wide over-expression of SSR corresponds to persistent differentiation and better prognosis, but the separate analysis of the subgroup of patients with stages III or IV in our series showed a non-significant trend for a better outcome only in patients with a high maxSUV $V_{\max }$. Overall, it appears that the cut-off value derived from Ambrosini et al. [3] may not be valid for DOTATOC, even in pNET stages III and IV.

We chose to measure volumes with a threshold of $41 \%$, as in the majority of FDG studies [9], but the pertinence of applying this arbitrary threshold to SRPET should be assessed by further studies. In the same way, in the absence of documented response criteria on SRPET, we used criteria adapted from RECIST to assess disease progression on ${ }^{68} \mathrm{Ga}$-DOTATOC. In particular, appearance of a new pathological focus meant disease progression. RECIST 1.1 criteria have been validated with FDG PET and their extension to SRPET was an innovation.

Disease progression was assessed on subsequent ${ }^{68} \mathrm{Ga}$-DOTATOC PET/CTs in 10 patients and on conventional imaging by $\mathrm{CT}$ or MRI in 7 patients. Follow-up ${ }^{68} \mathrm{Ga}$-DOTATOC PET/CTs for disease progression yielded independent information on PET/CT from the prognostic value of ${ }^{68} \mathrm{Ga}$-DOTATOC uptake by the primary tumor. We evaluated this in our study. The new pathological foci have been accepted as evidence for progression because this examination has a higher detection rate than conventional imaging in the detection of neuroendocrine pathological lesions [20].

To evaluate potential prognostic markers of pNET, we assessed the PFS rate at 24 months for two reasons: the median follow-up period in our series was 25.6 months and Ambrosini et al. [3] evaluated tumor progression at 24 months in the study on the prognostic value of maxSUV $V_{\text {max }}$. The PFS rate at 24 mo there was $47 \%$ compared to our $73 \%$, and this reflects our patients with lower grade.

Limitations to our study require consideration. As the volumetric approach had not been reported in literature for DOTATOC PET/CT in pNET, we had to perform ROC 
analyses to evaluate the best prognostic threshold of baseline TFTV to delineate patients who subsequently progress within $24 \mathrm{mo}$. We then used this cut-off value of $13.8 \mathrm{~cm}^{3}$ in univariate and multivariate analyses and this yielded overevaluated performance values. The actual prediction values using those cut-off values should be assessed in a validation study on an independent patient sample, because our study was designed to be a pilot study.

In conclusion, our study suggests that ${ }^{68} \mathrm{Ga}$-DOTATOC TFTV measured on PET images is a valuable prognostic biomarker in patients with well-differentiated pNETs in G1 and G2. A validation study is required to assess if a total functional tumor volume greater than $14 \mathrm{~cm}^{3}$ is indicative of high risk of progression within two years. This would have consequences on scheduling follow-up visits of those patients, as well as provide prognostic biomarkers and optimize the cost-effectiveness of monitoring those pNET patients.

Acknowledgements: We thank our clinician colleagues who referred the patients to our PET center, in particular Professor Philippe Ruszniewski and Doctor Eric Baudin. We acknowledge the excellent professional skills of our team of PET technologists. This study was carried out using data of patients from the prospective study “PHRC DOTATOC” P040303 EUDRACT 2007-002610-19, sponsored by Assistance Publique Hôpitaux de Paris (AP-HP). We would like to highlight the help of Zakia Idir from the direction of clinical research DRCD from AP-HP and of the clinical research assistants, Alexandra Kobetz from URC Lariboisière and others from CancerEst who committed themselves to gathering the follow-up data of patients.

\section{References}

[1] YAO JC, HASSAN M, PHAN A, DAGOHOY C, LEARY C et al. One hundred years after "carcinoid": epidemiology of and prognostic factors for neuroendocrine tumors in 35,825 cases in the United States. J Clin Oncol 2008; 26: 3063-3072. https://doi.org/10.1200/JCO.2007.15.4377

[2] PANZUTO F1, BONINSEGNA L, FAZIO N, CAMPANA D, PIA BRIZZI M et al. Metastatic and locally advanced pancreatic endocrine carcinomas: analysis of factors associated with disease progression. J Clin Oncol 2011; 29: 2372-2377. https://doi.org/10.1200/JCO.2010.33.0688

[3] AMBROSINI V, CAMPANA D, POLVERARI G, PETERLE C, DIODATO $S$ et al. Prognostic Value of 68Ga-DOTANOC PET/CT SUVmax in Patients with Neuroendocrine Tumors of the Pancreas. J Nucl Med 2015; 56: 1843-1848. https://doi. org/10.2967/jnumed.115.162719

[4] BOSMAN FT, CARNEIRO F, HRUBAN RH, THEISE ND (Eds.). WHO Classification of Tumors of the Digestive System. IARC, Lyon 2010, pp. 417. ISBN 9789283224327

[5] RINDI G, KLÖPPEL G, ALHMAN H, CAPLIN M, COUVELARD A et al. TNM staging of foregut (neuro) endocrine tumors: a consensus proposal including a grading system. Virchows Arch 2006; 449: 395-401. https://doi. org/10.1007/s00428-006-0250-1
[6] OBERG K. Gallium-68 somatostatin receptor PET/CT: is it time to replace (111)Indium DTPA octreotide for patients with neuroendocrine tumors? Endocrine 2012; 42: 3-4. https://doi.org/10.1007/s12020-012-9681-4

[7] SCHREITER NF, BRENNER W, NOGAMI M, BUCHERT R, HUPPERTZ A et al. Cost comparison of 111In-DTPAoctreotide scintigraphy and 68Ga-DOTATOC PET/CT for staging enteropancreatic neuroendocrine tumors. Eur J Nucl Med Mol Imaging 2012; 39: 72-82. https://doi.org/10.1007/ s00259-011-1935-5

[8] BYUN BH, KONG CB, PARK J, SEO Y, LIM I et al. Initial metabolic tumor volume measured by $18 \mathrm{~F}-\mathrm{FDG}$ PET/CT can predict the outcome of osteosarcoma of the extremities. J Nucl Med 2013; 54: 1725-1732. https://doi.org/10.2967/ jnumed.112.117697

[9] SASANELLI M, MEIGNAN M, HAIOUN C, BERRIOLO-RIEDINGER A, CASASNOVAS RO et al. Pretherapy metabolic tumor volume is an independent predictor of outcome in patients with diffuse large B-cell lymphoma. Eur J Nucl Med Mol Imaging 2014; 41: 2017-2022. https://doi. org/10.1007/s00259-014-2822-7

[10] LEE JW, KANG CM, CHOI HJ, LEE WJ, SONG SY et al. Prognostic Value of Metabolic Tumor Volume and Total Lesion Glycolysis on Preoperative (1)(8)F-FDG PET/CT in Patients with Pancreatic Cancer. J Nucl Med 2014; 55: 898-904. https://doi.org/10.2967/jnumed.113.131847

[11] EISENHAUER EA, THERASSE P, BOGAERTS J, SCHWARTZ LH, SARGENT D et al. New response evaluation criteria in solid tumors: revised RECIST guideline (version 1.1). Eur J Cancer 2009; 45: 228-247. https://doi. org/10.1016/j.ejca.2008.10.026

[12] BREEMAN WA, DE JONG M, DE BLOIS E, BERNARD BF, KONIJNENBERG $\mathrm{M}$ et al. Radiolabelling DOTA-peptides with 68Ga. Eur J Nucl Med Mol Imaging 2005; 32: 478-485. https://doi.org/10.1007/s00259-004-1702-y

[13] AIDE N, LASNON C, VEIT-HAIBACH P, SERA T, SATTLER B et al. EANM/EARL harmonization strategies in PET quantification: from daily practice to multicentre oncological studies. Eur J Nucl Med Mol Imaging 2017; 44: 17-31. https://doi.org/10.1007/s00259-017-3740-2

[14] LASNON C, ENILORAC B, POPOTTE H, AIDE N. Impact of the EARL harmonization program on automatic delineation of metabolic active tumor volumes (MATVs). EJNMMI Res 2017; 7: 30. https://doi.org/10.1186/s13550017-0279-y

[15] WEBER WA, ZIEGLER SI, THODTMANN R, HANAUSKE AR, SCHWAIGER M. Reproducibility of metabolic measurements in malignant tumors using FDG PET. J Nucl Med 1999; 40: 1771-1777.

[16] NILICA B, WAITZ D, STEVANOVIC V, UPRIMNY C, KENDLER D et al. Direct comparison of (68)Ga-DOTATOC and (18)F-FDG PET/CT in the follow-up of patients with neuroendocrine tumor treated with the first full peptide receptor radionuclide therapy cycle. Eur J Nucl Med Mol Imaging 2016; 43: 1585-1592. https://doi.org/10.1007/s00259016-3328-2 
[17] BAHRI H, LAURENCE L, EDELINE J, LEGHZALI H, DEVILLERS A et al. High prognostic value of $18 \mathrm{~F}-\mathrm{FDG}$ PET for metastatic gastroenteropancreatic neuroendocrine tumors: a long-term evaluation. J Nucl Med 2014; 55: 17861790. https://doi.org/10.2967/jnumed.114.144386

[18] GARIN E, LE JEUNE F, DEVILLERS A, CUGGIA M, DE LAJARTE-THIROUARD AS et al. Predictive value of $18 \mathrm{~F}$ FDG PET and somatostatin receptor scintigraphy in patients with metastatic endocrine tumors. J Nucl Med 2009; 50: 858-864. https://doi.org/10.2967/jnumed.108.057505

[19] KIM HS, CHOI JY, CHOI DW, LIM HY, LEE JH et al. Prognostic Value of Volume-Based Metabolic Parameters Measured by (18)F-FDG PET/CT of Pancreatic Neuroendocrine Tumors. Nucl Med Mol Imaging 2014; 48: 180-186. https:// doi.org/10.1007/s13139-013-0262-0
[20] GABRIEL M, DECRISTOFORO C, KENDLER D, DOBROZEMSKY G, HEUTE D et al. 68Ga-DOTA-Tyr3-octreotide PET in neuroendocrine tumors: comparison with somatostatin receptor scintigraphy and CT. J Nucl Med 2007; 48: 508-518.

[21] GABRIEL M, OBERAUER A, DOBROZEMSKY G, DECRISTOFORO C, PUTZER D et al. 68Ga-DOTA-Tyr3octreotide PET for assessing response to somatostatin-receptor-mediated radionuclide therapy. J Nucl Med 2009; 50: 1427-1434. https://doi.org/10.2967/jnumed.108.053421 